\title{
Neuromuscular Junction Transmission is Effected by Quantum Entanglement of Ion Transition States
}

\author{
M. Mostafizur Rahman ${ }^{1}$, Mufti Mahmud ${ }^{2}$ \\ ${ }^{1}$ NeuroChip Laboratory, University of Padova, Padova, Italy, rahman@dei.unipd.it \\ ${ }^{2}$ NeuroChip Laboratory, University of Padova, Padova, Italy, mahmud@dei.unipd.it; and Institute of Information \\ Technology, Jahangirnagar University, Dhaka, Bangladesh
}

\begin{abstract}
Neuromuscular Junction (NMJ) is the place where the neuron meets with muscle fiber crating a synapse to propagate the electrochemical signals. The infolds of the endplate of muscle fiber play a vital role containing a huge number of sodium channels in contraction of NMJ transmission mechanism. We have studied here the entanglement of sodium channel activation particle (' $\mathrm{m}$ ' particle). Through the simulation results we confirm that the entanglement of ' $\mathrm{m}$ ' particle effects the initiation time, amplitude and the rate of change of membrane potential.
\end{abstract}

\section{Introduction}

The junction between the terminal of a motor neuron and a muscle fiber where the neurons meets to ensure a rapid and efficient transmission of an action potential into depolarization of the postsynaptic target muscle by transmitting electro chemically excitation and causing the muscle to contract is called the Neuromuscular Junction (NMJ). The mechanism of neurotransmitter conduction has studied extensively [1-6]. At the NMJ the presynaptic neuron carries the impulse and the postsynaptic membrane undergoes a potential change eventually triggering the action potential. The opening of voltage-dependent calcium channels causes the release of neurotransmitters (ACh) that diffuse at the synaptic cleft and bind with Acetylcholine Receptors (AChR) causing inflow of sodium ions and outflow of potassium ions generating the end-plate synaptic potential (EPSP) at the post-synaptic membrane initiating an action potential [7].

The flow of current along the synaptic cleft $(15-200 \mathrm{~nm}$, [8]) affects the local extracellular potential, in turn affecting the voltage-dependent processes in the adjacent membranes. This phenomenon of current flow through weakly opened $\mathrm{Na}+$ channels causing potential drop at the extracellular space by boosting channels activation and positive feedback. According to this hypothesis, the drop of the voltage which is generated the cleft may modify the voltages across the muscle membrane which affects the conductance of voltagegated ion channels, thus increasing the probability of opening the voltage-gated ion channels. [9]

At NMJ a motoneuron meets muscle fibres creating a synapse to control the muscle contraction, in turn our movements. This electrochemical signal based communication takes place by activating and inactivating the ion channels at the junction's adjacent membranes. The large density of voltage gated sodium channels at the postsynaptic infolds plays the most vital role to stimulate the synaptic activation. $[7,8,10]$

The Hodgkin-Huxley Model (HHM) is the most widely accepted physiological model of single neuron. It describes the permissive and non-permissive states of the voltage gated ion channels and their mechanisms [11]. The classical HHM is a rate theory model that deal with gating particles of role playing ions. The rate theory model incorporating the atomic determination of the ion molecules may improvise the classical concept. Bernroider et al [12] has modified the classical HHM sodium ion gating probability to three similar but independent quantum correlated probabilistic mechanism where the sodium ion gating states during conduction are involved in entanglement of different degree of freedom. The incorporation of entanglement in sodium channel probability leads the gating mechanism slow down or rise.

In this work a realistic model mimicking the transmission process of NMJ was considered. This model takes into account the flow of current at junction due to the weakly opened sodium channels which affects the voltage-dependent processes at the adjacent membranes and boosts the postsynaptic membrane potential by a positive feedback. The sodium channel kinetics was calculated using the classical HHM using realistic physiological parameters. Entanglement of activation particles (m-gating) in terms of their quantum distribution was introduced using quantum theoretic approach which was adopted from Bernroider et al [12]. A current clamp simulation was performed to study the entanglement of $\mathrm{Na}+$ channel activation particle effects on action potential properties. The entangled relationship of m-gating particles changes the $\mathrm{Na}+$ channel activation by modifying sodium-ions activity in the voltage dependent ion-channels. Due to the change in the activation profile, the sodium current generated in the muscle endplate is changed which modifies the junction potential of the cleft. The subsequent result is the change of the cleft resistivity and action potential dynamics during the NMJ transmission process. 


\section{The model and parameters}

The NMJ was designed using a $50 \mu \mathrm{m}$ diameter and 500 $\mu \mathrm{m}$ long barrel shaped muscle fiber. Physiological parameters of rat NMJ were used in simulating the transmission process.

A circular axon terminal of $10 \mu \mathrm{m}$ radius $\left(\mathrm{a}_{\mathrm{j}}\right)$ [8] was connected to the muscle fiber membrane $\left(7.85 \times 10^{-8} \mathrm{~m}^{2}\right)$ to mimic the synapse. The maximum conductance $\left(\mathrm{g}_{\mathrm{jm}}\right)$ of voltage-gated sodium channels at the endplate was determined considering $9 \mathrm{pS} /$ channel [13] with a channel density of 3700 channels $/ \mu \mathrm{m}^{2}$ [14].

The potential at the neuromuscular junction cleft $\left(\mathrm{V}_{\mathrm{j}}\right)$ is calculated iteratively using equation 1 from the $G_{j m}$ and other contributors, such as $\mathrm{V}_{\mathrm{j}}$ itself, the sodium-specific reversal potential $\mathrm{V}_{0}(50 \mathrm{mV})$ [10], the global resistance of the cleft $\left(\mathrm{R}_{\mathrm{j}}\right)$ (eq. 5), the intracellular potential $\left(\mathrm{V}_{\mathrm{m}}\right)$ (Fig. 1).

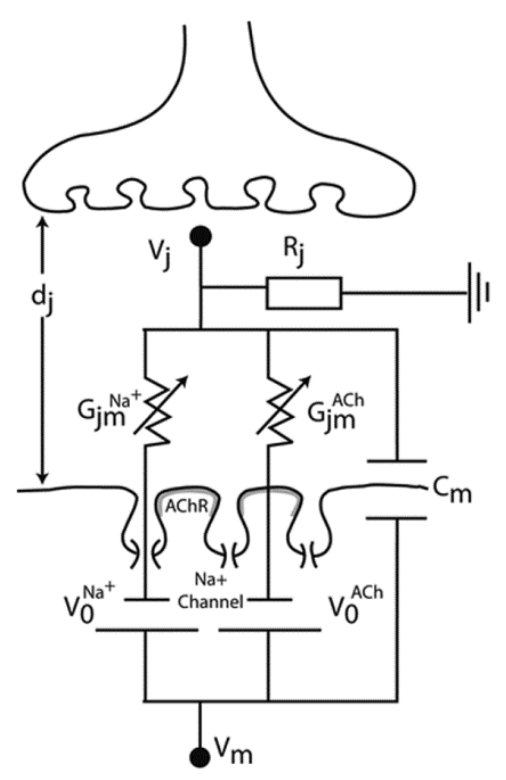

ig. 1. Equivalent electrical model of the NMJ. $R_{j}$, global resistance of the synaptic cleft; $\mathrm{d}_{\mathrm{j}}$, pre-to-postsynaptic membrane distance; $\mathrm{G}_{\mathrm{jm}}(\mathrm{AChR})$ and $\mathrm{G}_{\mathrm{jm}}\left(\mathrm{Na}_{\mathrm{v}}\right)$, global membrane conductances of AChR and $\mathrm{Na}_{\mathrm{v}}$ channels at the endplate with related reversal potentials $\mathrm{V}_{0}(\mathrm{AChR})$ and $\mathrm{V}_{0}\left(\mathrm{Na}_{\mathrm{v}}\right) ; \mathrm{C}_{\mathrm{m}}$, membrane capacitance; $\mathrm{V}_{\mathrm{m}}$, muscle fiber intracellular potential.

$V_{j+1}=G_{j m} \times R_{j} \frac{V_{m}-V_{j}-V_{0}}{1+\left(R_{j} \cdot G_{j m}\right)}$

The global sodium conductance $\left(\mathrm{G}_{\mathrm{jm}}\right)$ of the otoneuron and the sodium conductance $\left(\mathrm{g}_{\mathrm{jm}}\right)$ at the endplate are computed using eq. 2 and eq. 3 , respectively.

$\mathrm{G}_{\mathrm{jm}}=\mathrm{g}_{\mathrm{jm}} \cdot \pi \mathrm{a}_{\mathrm{j}}^{2}$

$\mathrm{g}_{\mathrm{jm}}=\overline{\mathrm{g}}_{\mathrm{jm}} \cdot \mathrm{m}^{3} \cdot \mathrm{h}$
The endplate sodium current $\left(\mathrm{EPI}_{\mathrm{Na}}\right)$ is calculated by eq.

4 , where $\mathrm{E}_{\mathrm{Na}}$ is the Nernst equilibrium potential.

$\mathrm{EPI}_{\mathrm{Na}}=\mathrm{G}_{\mathrm{jm}} \cdot\left(\mathrm{V}_{\mathrm{m}}-\mathrm{V}_{\mathrm{j}}-\mathrm{E}_{\mathrm{Na}}\right)$

The global resistance $R_{j}$ (eq. 5) is derived using cable equation and point contact model [9, 15-18].

$R_{j}=\frac{r_{j}}{z_{0}^{2} \pi}\left(\frac{24 z_{0}^{2}+2 z_{0}^{4}}{192+24 z_{0}^{2}+z_{0}^{4}}\right), z_{0}=a_{j} \sqrt{r_{j} * g_{j m}}, \mathrm{r}_{\mathrm{j}}=\frac{\rho_{\mathrm{j}}}{\mathrm{d}_{\mathrm{j}}}$

The AChR current $\left(\mathrm{I}_{\mathrm{AChR}}\right)$ (eq. 6), generated in the cleft is modeled as a double exponential function.

$I_{A C h R}=C_{j} \cdot V_{m} \cdot\left(\exp \left(-\frac{t}{\tau_{d}}\right)-\exp \left(-\frac{t}{\tau_{r}}\right)\right)$

$\mathrm{C}_{\mathrm{j}}$ is the total AChR conductance at the cleft (59.1 pS / channel [19]). The number of active AChRs is 10000 AChR channels $/ \mu \mathrm{m}^{2}[19]$. We considered $2 \%$ of the channels open at the time of synaptic activation with decay and rise time constants are $\tau_{\mathrm{d}}$ and $\tau_{\mathrm{r}}(0.564 \&$ $0.162 \mathrm{~ms}$, respectively [20]).

As shown in fig. 1, the $\mathrm{I}_{\mathrm{AChR}}$ together with the $\mathrm{EPI}_{\mathrm{Na}}$ act as stimulus current (eq. 7) to carry out the HodgkinHuxley speculation for triggering an action potential (eq. 8).

$\mathrm{I}_{\mathrm{stim}}=\mathrm{I}_{\mathrm{AChR}}+\mathrm{EPI}_{\mathrm{Na}}$

$\mathrm{V}_{\mathrm{m}}=-1 / \mathrm{C}_{\mathrm{m}} \cdot\left(\mathrm{I}_{\mathrm{Na}}+\mathrm{I}_{\mathrm{K}}+\mathrm{I}_{\mathrm{L}}+\mathrm{I}_{\text {stim }}\right)$

Where the membrane capacitance $C_{m}$ is $4 \mu \mathrm{F} / \mathrm{cm}^{2}$ [14].

The sodium, potassium and leakage current are calculated using classical Hodgkin-Huxley formalism [11] using $\overline{g_{N a}}\left(45 \mathrm{mS} / \mathrm{cm}^{2}\right.$ [10]), $\overline{\mathrm{g}_{\mathrm{K}}}\left(29.9 \mathrm{mS} / \mathrm{cm}^{2}\right.$ [21]), $\overline{\mathrm{g}_{\mathrm{L}}}\left(1 \mathrm{mS} / \mathrm{cm}^{2}\right)$ as the maximum conductances, and $\mathrm{E}_{\mathrm{Na}}(45 \mathrm{mV}[10]), \mathrm{E}_{\mathrm{K}}\left(-73 \mathrm{mV}\right.$ [21]), $\mathrm{E}_{\mathrm{L}}(-90 \mathrm{mV}$ [14]) as the reversal potentials for sodium, potassium, and leakage, respectively.

Adoprting the quantum entanglement of sodium ion activation particle from Bernroider et al [12] the degree of freedom of ' $m$ ' particle is redifned as

$0 \leq \delta^{2} \leq \frac{1}{1-3 m+3 m^{2}}$

To have the quantum mechanical correction for the HHM, the term ' $m^{3}$ ' is replaced by the term $\boldsymbol{\delta}^{2} \mathrm{~m}^{3}$. Another variable ' $k$ ' is incorporated to control the degree of freedom. $k=0$ is the classical HHM condition without any entanglement and the positive values of $k$ results an increased conduction rate of sodoim channela. $k=1$ is the maximum positive entanglement with $\delta^{2}=\frac{1}{1-3 m+3 m^{2}}$. In the case of negative entanglement the term $k$ is defined as $\boldsymbol{k}:=\boldsymbol{\delta}^{\mathbf{2}}-1$.So,

Case I: if $\quad k \geq 0, \quad \boldsymbol{\delta}^{2}=1+k \frac{3 m(1-m)}{1-3 m(1-m)}$

Case II: if $k<0, \quad \delta^{2}=1+k$ 


\section{Simulated results and discussion}

Voltage-clamp experiments were simulated in MATLAB through scripting (version: R2009b; www.mathworks.com).

At the rat $\mathrm{NMJ}$ to simulate the action potential $400 \mathrm{nA} / \mathrm{cm}^{2}$ constant curret was applied for differnt $k$ (fig 2). For $\mathrm{k}=0$ (classical HHM situation) it takes $2.9 \mathrm{~ms}$ to reach the threshold of $-57.37 \mathrm{mV}$ to initiate the action potrential and reaches the maximum of $35.5 \mathrm{mV}$ at 3.75 $\mathrm{ms}$. Where as for $\mathrm{k}=1$ (maximum positive entanglement) it reaches the threshod $(-60.66 \mathrm{mV})$ at $2.665 \mathrm{~ms}$ and maximum $(39.5 \mathrm{mV})$ at $3.285 \mathrm{~ms}$.

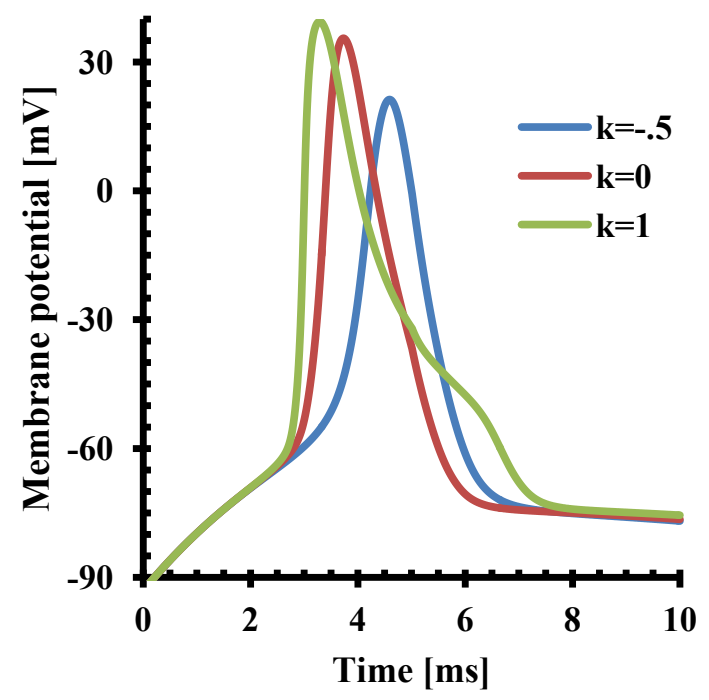

Fig 2: Action potential generated at the NMJ for different value of $\mathrm{k}$.

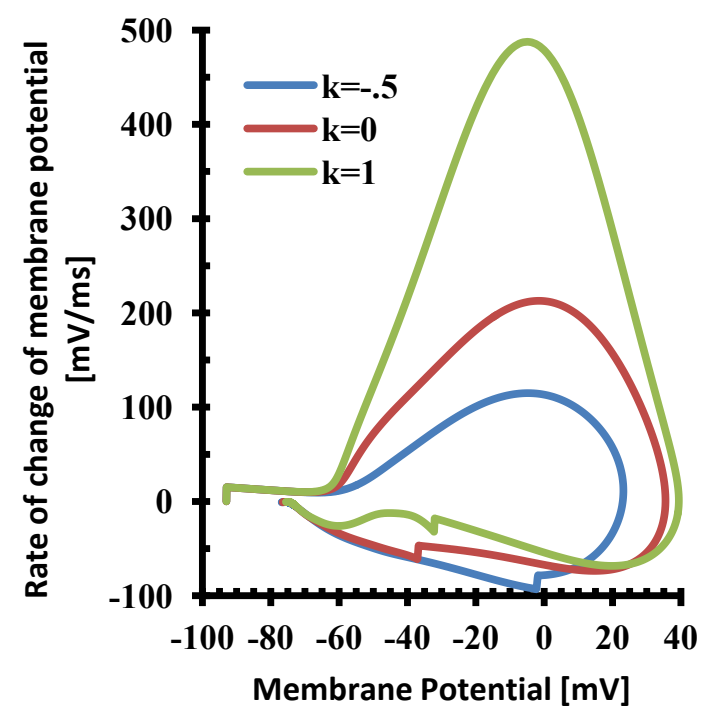

Fig 3: Phase plot for different value of $\mathrm{k}$.

Figure 3 is showing the phase plot of the rate of change of membrane potential. For maximum positive entanglement $(\mathrm{k}=1)$ the maximum rate of rise of action potential is $487.4 \mathrm{mV} / \mathrm{ms}$. In the classical HHM case $(\mathrm{k}=0)$ this is $212 \mathrm{mV} / \mathrm{ms}$.

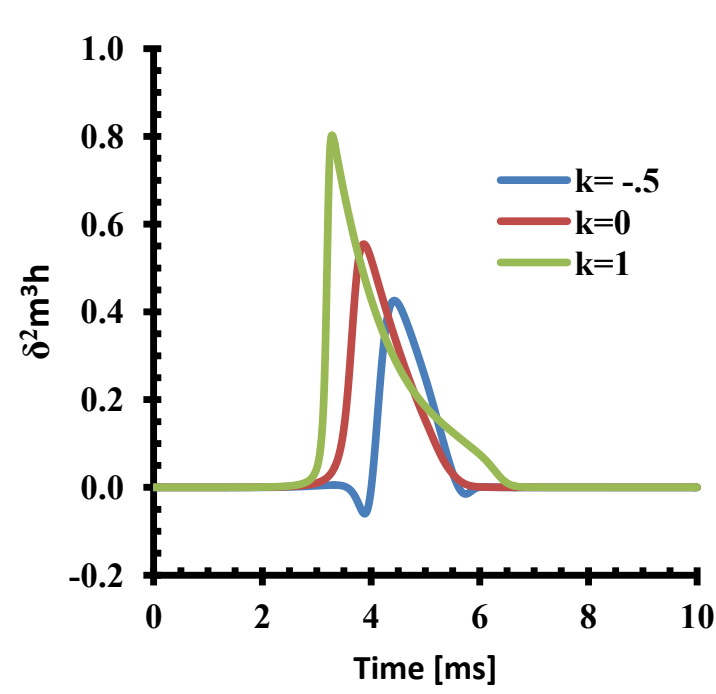

Fig 4: Resulting probability $\left(\boldsymbol{\delta}^{\mathbf{2}} m^{3} h\right)$ of sodium channel gating kinetics for different value of $k$.

According to the classical HHM, the sodium channel contains three identical, rapidly responding, activation gates of type ' $m$ ' and a single, slower responding, inactivation gate of type ' $h$ '. Therefore, $m^{3} h$ is the resultant probability of the sodium channel opening at any time instant. In our case incorporating the entanglement of the activation gates of type ' $m$ ' the resultant probability is $\boldsymbol{\delta}^{2} m^{3} h$. Figure 4 is shoing the resultant probability of the sodium channel gating kinetics for different degree of freedom. At $k=0$, the maximum resultant probability is 0.5724 at $3.62 \mathrm{~ms}$ where as this is 0.814 at $3.08 \mathrm{~ms}$ for $k=1$. And for negative entanglement the maximum resultant probability is 0.414 at $4.45 \mathrm{~ms}$.

From this simulation results it is clear that in the case of maximum positive entanglement the action potential initiates at a 5\% less threshold value and to reach the peak amplitude it takes $13.5 \%$ less time than classical HHM condition. For $\mathrm{k}=-0.5$ it is showing the reverse effect. The rate of change of membrane potential simulation is also shoing the similar concequences. From the resultant probability simulation it show that in the case of maximum positive entanglement it is $17.5 \%$ faster and $42.3 \%$ more activation than the classical HHM case.

The earlier and faster onset at positive entanglement condition in principle is exhibiting the higher reliability and more efficiency of transmission mechanism. The entangled relationship of $\mathrm{m}$-gating particles changes the $\mathrm{Na}+$ channel activation by modifying sodium-ions activity in the voltage dependent ion-channels. Due to the change in the activation profile, the sodium current generated in the muscle endplate is changed which modifies the junction potential of the cleft. The subsequent result is the change of the cleft resistivity and action potential dynamics during the NMJ transmission process. 


\section{Conclusion}

Till today the Hodgkin-Huxley model is the most biophysically realistic and meaningfull model to mockup from a single neuron to a large neural network. The voltage-dependent ion-channels cause an action potential to be initiated. According to the classical HHM, the sodium channel contains three identical, rapidly responding, activation gates of type ' $\mathrm{m}$ '. In this work, we have presented a realistic NMJ model taking into account the quantum entanglement of the activation particles that modifies the channel kinetics, in turn the transmission process of the NMJ. This effect may play a role in designing efficient artificial NMJ for neuroprosthetic applications.

\section{$5 \quad$ References}

[1] Hoch, W.: Formation of the neuromuscular junction : Eur. J. Biochem., Vol. 265, pp. 1-10, 1999.

[2] Sanes, J. R., Lichtman, J. W. : Development of the Vertebrate Neuromuscular Junction : Annu. Rev. Neurosci., Vol. 22, pp. 389-442, 1999.

[3] Davis, G., Goodman, C. : Genetic analysis of synaptic development and plasticity: homeostatic regulation of synaptic efficacy: Curr. Opin. Neurobiol., Vol. 8, pp. 149-156, 1998.

[4] Thomas, M., Bruce, G.: Formation of the neuromuscular junction: molecules and mechanisms: Bioessays, Vol. 20, pp. 819-829, 1998.

[5] Deschenes, M.R. , Maresh, C.M., Kraemer, W.J. : The neuromuscular junction structure, function, and its role in the excitation of muscle: J. Strength Cond. Res., Vol. 8, pp. 103-109, 1994.

[6] Zach, W., Joshua, R. : Synaptic structure and development: the neuromuscular junction : Cell, Vol. 72, pp. 99-121, 1993.

[7] Martin, A. : Amplification of neuromuscular transmission by postjunctional folds : Proc. R. Soc. B, Vol. 258, pp. 321-326, 1994.

[8] Wood, S. J., Slater, C. R. : Safety Factor at the Neuromuscular Junction : Prog. Neurobiol., Vol. 64, No. 4, pp. 393-429, 2001.

[9] Fromherz, P. : Self-Gating of Ion Channels in Cell Adhesion : Phys. Rev. Lett., Vol. 78, No. 21, pp. 4131--4134, 1997.

[10] Desaphy, J. F., De Luca, A., Imbrici, P., Camerino, D.C. : Modification by Ageing of the Tetrodotoxin-Sensitive Sodium Channels in Rat Skeletal Muscle Fibres : Biochim. Biophys. Acta., Vol. 1373, No. 1, pp. 37-46, 1998.

[11] Boudier, J.L., LeTreut, T., Jover, E. : Autoradiographic localization of voltagedependent sodium channels on the mouse neuromuscular junction using 125 alpha scorpion toxin. II. Sodium channel distribution on postsynaptic membranes : J. Neurosci., Vol. 12, No. 2, pp. 454-466, 1992.

[12] Villarroel, A., Sakmann, B. : Calcium permeability increase of endplate channels in rat muscle during postnatal development : J. Physiol., Vol. 496, No. 2, pp. 331-338, 1996.

[13] Head, S.D. : Temperature and endplate currents in rat diaphragm : J. Physiol., Vol. 334, pp. 441-459, 1983.

[14] Pappone, P. A. : Voltage clamp experiments in normal and denervated mammalian skeletal muscle fibers : J. Physiol., Vol. 306, pp. 377-410, 1980.

[15] Hodgkin A. L., Huxley, A. F. : A quantitative description of membrane current and its application to conduction and excitation in nerve : J. Physiol., Vol. 117, pp. 500-544, 1952.

[16] Hollingworth, S., Marshall, M. W., Robson, E. : Ionic currents and charge movements in organcultured rat skeletal muscle : J. Physiol., Vol. 357, pp. 369-386, 1984.

[17] Bernroider, W., Summhammer, J. : Can Quantum Entanglement Between Ion Transition States Effect Action Potential Initiation ? : Cogn. Comput., Vol. 4, pp. 29-37, 2012.

[18] Fromherz, P. , Muller, C.O. , Weis , R. :Neurontransistor: electrical transfer function measured by the patch-clamp technique: Phys Rev Lett, Vol. 71, pp. 4079-4082, 1993.

[19] Weis, R., Fromherz, P. :Frequency dependent signal-transfer in neuron-transistors: Phys Rev E, Vol. 55, pp. 877-889, 1997.

[20] Schatzthauer, R. , Fromherz, P. : Neuron-silicon junction with voltage-gated ionic currents: Eur J Neurosci, Vol. 10, pp. 1956-1962, 1998.

[21] Vassanelli, S., Fromherz, P. : Transistor-records of excitable neurons from rat brain: Appl Phys A, Vol. 66, pp. 459-463, 1998. 